\title{
Perspectivas para la intervención en lo social desde los retos del proceso de paz colombiano ${ }^{1}$
}

\section{Prospects for intervention in the social from the challenges of colombian peace process}

\author{
Fernanda Torres-Gómez ${ }^{2}$
}

\begin{abstract}
Resumen
Ante el panorama actual de implementación de los acuerdos de paz de la Habana, la sociedad colombiana atraviesa un momento histórico de esperanza e incertidumbre, en el cual es prioritario re-pensarnos la intervención en lo social. El presente artículo tiene como objetivo presentar un conjunto de perspectivas y desafíos para la intervención desde la socio-terapia con enfoque de acción sin daño, producto de un ejercicio de investigación cualitativa, con enfoque hermenéutico, bajo la modalidad de investigación documental y análisis de contenido, que plantea la reflexión del ejercicio profesional en la reparación integral de víctimas en el Distrito Capital. Como propuestas de hallazgos se plantea la importancia de re-significar los impactos del conflicto armado desde intervenciones profesionales éticopolíticas, en las que se aborde el manejo de las emociones, lo que permitirá contribuir a garantizar los derechos de todos los ciudadanos, movilizando sus recursos y capacidades, desde la perspectiva de construcción democrática de la paz. Se identifica la importancia de pensar el lugar del autocuidado y la reflexividad del profesional en los procesos de intervención con víctimas, debido a los riesgos emocionales que genera este escenario social.
\end{abstract}

Palabras clave: Intervención profesional; socio-terapia; acción sin daño; reparación integral; conflicto armado interno

\begin{abstract}
Given the current panorama of implementation of the Havana Peace Agreements, Colombian society is going through a historic moment of hope and uncertainty, in which it is a priority to rethink our intervention in the social sphere. This article aims to present a set of perspectives and challenges for the professional intervention from the socio-therapy with action without damage, product of a qualitative research exercise, with a hermeneutical approach, under the modality of documentary research and analysis of content, which raises the reflection of the
\end{abstract}

Tipología: Artículo de reflexión

Recibido: $22 / 03 / 2016$

Evaluado: $19 / 01 / 2017$

Aceptado: 23/01/2017

Disponible en línea: 01/03/2017

Como citar este artículo: Torres-Gómez, F. (2017). Perspectivas para la intervención profesional en lo social desde los retos del proceso de paz colombiano. Jangwa Pana, 16 (1), 112 - 121. Doi: http://dx.doi.org/10.21676/16574923.1961

1. Artículo de reflexión que hace parte del proyecto de investigación titulado: "Solidaridad y Confianza en los programas de reintegración de excombatientes y atención psicosocial a víctimas en los escenarios de transición política en Colombia", vinculado al Centro de Estudios en Desarrollo y Territorio -CEDT- Vicerrectoría de Investigación y Transferencia VRIT. Universidad de la Salle.

2. M.Sc. en Docencia. Esp. Consultoría en familia y redes sociales. Trabajadora Social. Docente investigadora de la Universidad de La Salle, Bogotá, Colombia. Correo electrónico: mftorres@unisalle.edu.co 
professional practice in the integral reparation of victims in the Capital District (Bogotá). The findings suggest that it is important to re-signify the impacts of armed conflict from ethical-political professional interventions that address the management of emotions. This will help to guarantee the rights of all citizens by mobilizing their resources and will increase the possibility of democratic building of peace. The importance of thinking about the place of the self-care and reflexivity of the professional in the processes of intervention with victims is identified because of the emotional risks generated by this social scenario.

Keywords: Professional intervention; socio- therapy; action without damage; comprehensive repair; internal armed conflict

\section{Introducción}

\section{Acercamiento al contexto y sus retos}

$\mathrm{E}^{\mathrm{I}}$ 1 conflicto armado en Colombia se configura como uno de los conflictos internos más antiguos de la historia reciente del país. Se caracteriza por su complejidad, su trayectoria y los impactos particulares que han dejado huella desde hace más de sesenta años en la memoria individual y colectiva del pueblo colombiano. Según cifras del informe "¡Basta ya!” (CNMH) del año 2013, entre 1985 y 2012 Colombia tuvo un total de 5.712 .506 desplazamientos forzados, 27.023 secuestros en el periodo entre 1970 y 2012, 25.077 desapariciones forzadas entre 1985 y 2012 , y en ese mismo periodo de tiempo 10.189 víctimas de minas antipersona y 1.982 masacres. Como resultado de todo esto, entre 1985 y 2012, hubo 177.307 muertes de civiles, frente a 40.787 muertes de combatientes.

En este complejo escenario, quienes han padecido directamente las consecuencias de este flagelo han sido tradicionalmente las víctimas, quienes se ven impactadas en sus esferas microsociales y macrosociales, con consecuencias como las citadas por Cifuentes (2005): rupturas en el tejido social como producto de la desconfianza en las relaciones próximas; transformaciones en las estructuras y dinámicas psicosociales, ambientales, culturales y familiares como producto de los hechos victimizantes como desaparición forzada, secuestros, desplazamiento forzado, reclutamiento en grupos al margen de la ley, entre otros.
Este panorama plantea un reto trascendental referido a la reparación integral de las víctimas del conflicto armado interno, como eje central en el proceso político que permitirá la implementación de los seis puntos del acuerdo de paz firmado entre el gobierno y la guerrilla de las FARC. Esta implementación, fundada sobre el marco legal de la justicia transicional, esboza como escenarios posibles brindar a las FARC un estatus como movimiento político, condicionado al abandono de las armas; la no inmunidad a crímenes de lesa humanidad que serían castigados con restricción de la libertad durante entre 5 y 8 años; la restauración y reparación a las víctimas mediante la creación de la jurisdicción especial para la paz, bajo el principio de la justicia como respeto por la institucionalidad y la legislación en coherencia con la Constitución Nacional, el Derecho Internacional Humanitario, y los principios de justicia, verdad, reparación y no repetición.

Frente a las incertidumbres y desafíos que plantea el acuerdo de paz, nociones como justicia transicional, reconciliación y reparación integral adquieren nuevos significados y obligan a gestar nuevas rutas en torno a la investigación e intervención en el marco del conflicto armado con el fin de evidenciar las diferentes dimensiones y esferas de actuación de los sujetos víctimas, inmersos en construcciones sociales y relacionales complejas, en el marco del posacuerdo en nuestro país, teniendo en cuenta las particularidades de nuestro conflicto armado: multiproblémico, polifacético y multicausal. (Torres, 2013). En medio de esta situación, la sociedad civil —que vincula actores como la academia - tiene como 
reto potenciar la consolidación de la paz que tanto anhela nuestro país, a partir de la formación integral de profesionales que incidan en la configuración de nuevas formas de relaciones democráticas, donde todos los ciudadanos y ciudadanas tengan la posibilidad de expresarse y participar sin importar su edad, su género, su religión, entre otras, para reconstruir los vínculos que poseen en la perspectiva de garantía y promoción de los derechos humanos en torno a la convivencia, el respeto y la tolerancia.

En este horizonte, surge la necesidad de reflexionar sobre el papel que desempeñan los profesionales que intervienen con víctimas en el marco de conflicto armado interno, para que estén en capacidad de proyectar los desafíos que traerá el proceso de paz en Colombia, a partir del posacuerdo. A esto pretende contribuir el presente artículo de reflexión que propone un conjunto de perspectivas teóricometodológicas sobre la intervención en lo social, desde la óptica de la socio-terapia con enfoque de acción sin daño, cuyo eje gira en torno a la dignidad humana y al ejercicio de la ciudadanía, para apoyar la acción propia de los sujetos sociales desde la intervención en lo social. Esta propuesta es producto de un ejercicio de investigación en torno a una comprensión situada (Pineda \& De Alvarado, 2008) de categorías como la intervención profesional, la reparación integral, y las víctimas. En esa indagación, reconstruimos sus significados e implicaciones en los documentos que condensan la ley de víctimas y restitución de tierras, ley 1448 de 2011, y que fueron implementados por el Distrito Capital (Bogotá) frente a la reparación integral en el 2015. Entre esos documentos se ubica el Plan de desarrollo Bogotá Humana 2012-2016, el Programa de prevención, asistencia, atención, protección y reparación integral a las víctimas, el Plan de acción Distrital de Bogotá para la atención y reparación integral a las víctimas, y el protocolo de participación efectiva de las víctimas del conflicto armado para Bogotá, entre otros.

En este orden de ideas, la investigación desarrollada fue de tipo cualitativa es decir, utilizó "la recolección de datos sin medición numérica para descubrir o afinar preguntas de investigación en el proceso de interpretación" (Sampieri, 2010, p.9).
En esta lógica, la investigación asumió un enfoque hermenéutico, el cual según Vasco (1990) "trata de dar una interpretación global a un hecho, de comprenderlo, de darle el sentido que tiene para el grupo que está comprometido en esa praxis social" (p.14). La modalidad de investigación abordada en el presente ejercicio fue la investigación documental, como una alternativa que propende por recuperar los significados de los textos a partir de la interpretación y análisis de la información consignada en las fuentes documentales vinculadas a planes, programas y proyectos que adoptan en Bogotá el Plan Nacional de atención y reparación integral a las víctimas. Finalmente, como método de investigación, se asumió el análisis de contenidos, debido a que es una herramienta que permite comprender la complejidad de la realidad social (Ruiz, 2006).

\section{Discusión}

\section{Horizontes de la intervención en lo social}

En este apartado se perfilan las ideas centrales respecto a la perspectiva de intervención en lo social, producto del ejercicio de revisión documental y análisis de contenido realizado a los documentos que orientan la reparación integral a víctimas en el Distrito Capital. Tales ideas centran su atención en la propuesta de la socio-terapia, el enfoque de acción sin daño fundado en la confianza, la escucha y el perdón como los principales derroteros teóricometodológicos que permitan superar la noción tradicional de intervención profesional desde el paradigma tradicional-positivista que, retomando a Morán Carrillo (2007), centra su atención en procesos con perspectiva causal, instrumental, aséptica en valores, limitada a un campo de conocimiento que reduce la posibilidad de construir y transformar desde lo interdisciplinar. El tránsito de conceptos implica resignificar la intervención en lo social en el escenario que traerá el posacuerdo, reconociendo cómo el Estado y la academia, específicamente desde las ciencias sociales, estamos convocados a participar en la transformación de los 
imaginarios y representaciones sociales, mediante la reconstrucción del tejido social y la incidencia en diferentes espacios en pro del reconocimiento legítimo de los derechos de las víctimas, y mediante prácticas que favorezcan la reconciliación, la escucha, el perdón y la confianza, con lo que se puedan construir nuevas relaciones y abordajes de manejo del conflicto no violentas, democráticas y pacíficas.

Lo anterior nos lleva a transitar hacia comprensiones e intervenciones alternativas de orden psico-sociojurídicas del conflicto armado, la justicia y la reparación integral, y del papel que tenemos en la configuración de nuestro campo de conocimiento, contemplando:

Una dificultad de diálogo entre lo social, las nuevas formas de padecimiento, el deseo, la memoria colectiva en su expresión singular y el acceso a los escenarios de intervención social con mayor certeza y conocimiento profundo de ese otro sobre el que se interviene, tanto desde lo individual como desde lo colectivo. (Carballeda, 2010, p.48)

De este modo podemos marchar hacia un nuevo lugar de intervención en lo social, desde miradas contextualizadas, analíticas, integrales y éticopolíticas, que superan las nociones tradicionales reduccionistas de nuestro saber-hacer propuestas en programas, planes y proyectos referidos a víctimas del conflicto armado en Colombia, expresadas por ejemplo en procesos terapéuticos individuales que desligan al sujeto de su contexto familiar y comunitario; intervenciones que homogenizan las identidades de las víctimas del conflicto armado en un estándar de vulnerabilidad sesgado por imaginarios ante el deber ser de la reparación integral como figura jurídico-administrativa, y la noción inmediatista de la acción profesional, sin una fundamentación interdisciplinar. Es por ello pertinente transitar hacia la socio-terapia como propuesta de intervención en lo social que brinda una mirada holística de la realidad, promoviendo análisis de las diferentes dimensiones del sujeto que han sido impactadas por el conflicto armado, con el fin de co-construir alternativas de trasformación que reconozcan la emocionalidad, la racionalidad y la generatividad.

En este sentido, Carballeda (2002) plantea que la intervención es un proceso que actúa y hace actuar, que produce expectativas y consecuencias que deben ser reconocidas y abordadas por el profesional. Así, "la intervención implica una inscripción en ese 'otro' sobre el cual se interviene, quien a su vez genera una 'marca' en la institución y desencadena una serie de dispositivos e instrumentos en esta" (p. 94). Este horizonte ubica la intervención en un entramado de relaciones y un conjunto de tensiones denominado "lo social", configurado como entorno de interacción en el cual se objetiva la realidad social en forma de problemas o tensiones sociales, que involucran la ruptura en dichas situaciones de los papeles asignados al Estado, el mercado y la sociedad civil, en el marco del modelo económico, político, ambiental y cultural patriarcal-capitalista predominante, que en el caso colombiano está matizado, por crisis estructurales como la pobreza, la corrupción, la injusticia, la exclusión social y el conflicto armado interno. (Rozas, 2001).

Esta concepción de lo social lo entiende como una producción del ser humano, ya que se trata de una construcción conversacional conjunta con sus formaciones socio-cultural y psicológica, que transcurren en la vida cotidiana, desde la realidad y subjetividad de cada actor (Berger \& Luckmann, 2003). Por tanto, en este escenario surge la intervención en lo social como estrategia que posibilita descubrir en las víctimas afectadas por la violencia socio-política un repertorio de posibilidades, capacidades y recursos que les permitan re-significar su experiencia de dolor y sufrimiento. Indispensablemente, y siguiendo los postulados de Camps (2011), hay que reconocer aquí el papel que desempeñan las emociones en las relaciones intersubjetivas, toda vez que es imposible concebir a los sujetos sin sus dimensiones emocional y racional, las cuales contribuyen a movilizar o paralizar el actuar.

Es por ello vital que el profesional reconozca el entramado socio-ecológico que rodea a los sujetos involucrados en la socio-terapia: sus familias, 
sus redes sociales, comunitaria e institucionales, y que promueva desde allí un escenario de intervención en permanente construcción, a la luz de epistemologías generativas orientadas por la permanente autorreflexión, encaminadas a comprender las dimensiones territorial, contextual y simbólica de la cotidianidad del conflicto de modo que su abordaje pueda superar las puntuaciones y observaciones fragmentadas, asépticas y aisladas.

Una lectura crítica de la intervención profesional en lo social implica reconocer la complejidad de los escenarios sociales y los campos que desde allí se construyen, puesto que dicho proceso, según Alayón (2007), no se consolida como una categoría en abstracto. Por el contrario, requiere una postura ético-política coherente con las determinaciones histórico-sociales que caracterizan nuestros países latinoamericanos, en coyunturas particulares que requieren nuestra actuación profesional, una actuación que se cuestione sobre el por qué, el cómo y el para qué de nuestras intencionalidades disciplinares.

Desde esta perspectiva, la intervención profesional debe respaldarse en argumentos epistemológicos, teóricos y metodológicos que revaloricen el lugar de la formación profesional en la transformación de las realidades sociales injustas que vulneran los derechos humanos, por lo cual se deben construir, como lo indica Corvalán (1996):

Intervenciones pertinentes, relevantes y significativas que incida en la especificidad profesional, que se comprendan desde "el carácter socio-político de una intervención social [...] dado por la concepción de la misma en torno a objetivos societales mayores $\mathrm{y}$ relacionados con el funcionamiento del modelo de desarrollo de una sociedad (p. 4).

Desde esta configuración, la socio-terapia, según Calvo (2013), se concibe como estrategia de intervención en lo social que contempla una lectura analítica y reflexiva de los contextos sociales, culturales, económicos entre otros, de los sujetos que acuden a los profesionales en búsqueda de alternativas para modificar las manifestaciones de los problemas sociales que padecen, desde los cuales es clave interpretar las creencias, conceptos y prácticas que rodean la cultura y comunicación en dichos contextos. Atender a estas particularidades moviliza la transformación multidimensional, contemplando la riqueza del escenario de intervención de la atención psicosocial, que suele ser reducido en algunas instituciones y programas, como se mencionó previamente, a una estrategia caracterizada por: atender exclusivamente los impactos psicológicos generados por el trauma de padecer las consecuencias del conflicto armado, homogenizar los impactos diferenciales de cada hecho victimizante, y coartar las posibilidades de movilización y empoderamiento político del sujeto en su entorno.

La socio-terapia parte de una fundamentación epistemológica interdisciplinar que introduce la mirada psico-socio-jurídica de la intervención profesional en escenarios de reparación integral, con lo cual se co-construyen nuevas narrativas y diálogos generativos, cimentados en la confianza, la solidaridad, la escucha activa, la reconciliación y el perdón, que aportan a la re-significación de las dinámicas y realidades que producen los hechos victimizantes. Gracias a la introducción de esos criterios, se configuran nuevos relatos de vida a partir del enfoque de acción sin daño, donde se abordan históricamente las huellas psicosociales y simbólicas en lo individual, familiar y colectivo, producto de la violencia en sus diversas expresiones (Bello, 2013).

La intervención en lo social remite entonces a un proceso permanente de reflexividad, interpretada por Mosquera (2011) como una actividad que emerge de los saberes de acción, como interconexión de la emoción y la razón. La reflexividad ante los impactos que produce nuestra acción como profesionales que intervienen en lo social, se articula filosóficamente con el enfoque de la acción sin daño, cuyo origen se remonta a la discusiòn en torno a las consecuencias e impactos negativos que generaban, como actores externos, las intervenciones de carácter humanitario de los años noventa en países con conflictos armados, puesto que según, Rodríguez (2008), en algunos casos la intervención funda acciones que mitigan 
impactos y daños, al movilizar estrategias de abordaje alternativo y pacífico del conflicto, y en otras, desafortunadamente genera rupturas y daños como fuente externa.

Por lo anterior, el enfoque de la acción sin daño va de la mano del enfoque de derechos, toda vez que reconoce la capacidad de agencia de los sujetos como actores sociales, para re-construir y significar su experiencia frente al conflicto, a partir de la mediación del otro profesional, capaz de aportar a la reparación integral a la luz de una lectura compleja del contexto socio-cultural y simbólico de las víctimas, vinculando como lo menciona Rodríguez (2008), la construcción de paz desde la autonomía y la búsqueda de recursos, movilizando en el sujeto profesional que interviene en lo social: la autocrítica, la capacidad de asombro, de aprendizaje y de aporte al bienestar integral.

Al interior de la reflexión de la intervención en lo social, desde la socio-terapia, aparece la confianza como un derrotero de acción profesional, reconociéndola como un principio rector de las relaciones entre humanos, donde se establecen actitudes de reconocimiento y respeto de las ideas, sentimientos, imaginarios, posturas de los otros como sujetos en equidad de condiciones, a partir de su legitimidad como interlocutor en la convivencia, cimentada en la percepción de certeza que plantea Vives (2015), en el transcurso de un tiempo determinado. Lo anterior implica entregarse al otro, asumiendo el riesgo de equivocarse o ser engañado, lo que requiere apertura al otro en su diversidad, al mismo tiempo que la posibilidad de asumir un riesgo en la construcción de paz, puesto que la desconfianza obstaculiza la escucha, en la medida en que cada actor dejará de escuchar al otro, a quien va a deslegitimar, y en cuyos argumentos no va a creer porque los considerará un engaño.

En sintonía con la confianza, surgen la escucha y el perdón. La primera de estas como actitud (Vives, 2015), realizada desde nuestra subjetividad, nuestro contexto, cultura, emociones, historia y experiencia de vida, que atribuye un significado particular al tiempo-espacio que construye cada sujeto desde su trama relacional, lo cual condicionará su actitud frente a los otros y sus argumentos en pro del diálogo. La segunda, el perdón, como acto liberador que permite la garantía de no repetición, posibilita el reconocimiento público y la re-significación de la experiencia victimizante, hacia un camino que transforma el dolor simbólico de la pérdida y el duelo.

\section{Conclusiones}

\section{Perspectivas en la construcción de paz}

A partir de la investigación documental realizada, y teniendo como marco el fundamento epistemológico de la socio-terapia, como lo hemos expuesto, es menester perfilar algunas perspectivas desde la intervención en lo social hacia la búsqueda de la reparación integral a víctimas del conflicto armado interno. Las conclusiones presentadas pretenden provocar reflexiones epistemológicas sobre el momento histórico al que nos enfrentamos los profesionales de las ciencias sociales, como investigadores y gestores de la transformación social de nuestro país, en la vía de construir una paz imperfecta, término acuñado por Muñoz (2001), que trasciende las nociones de paz positiva y paz negativa, como ideales utópicos expresados por ausencia de conflictos de diferente orden. En este sentido, desde la socio-terapia con enfoque de acción sin daño, las orientaciones de intervención en lo social son:

- Comprender de forma holística la reparación integral de las víctimas del conflicto armado interno, como escenario vital de coresponsabilidad a largo plazo para la construcción de nuevas ciudadanías, desde el fortalecimiento de la socialización democrática y la ética del cuidado: la primera de éstas relacionada con la posibilidad de fundar desde la familia, la escuela y la comunidad nuevas relaciones participativas y pacíficas para afrontar los conflictos de la cotidianidad. La ética del cuidado, por su parte, referida a un actuar que reconoce y se preocupa por la dignidad del otro, como sujeto que comparte mi ejercicio de ciudadanía. 
Ahondar en estos pilares invita a contemplar el posicionamiento de la perspectiva de reparación moral, simbólica y material, que debe traer el posacuerdo, como apuesta para superar la reducción a la satisfacción económica que se ha otorgado al derecho de reparación que poseen las víctimas, puesto que dar prioridad a lo material deja de lado la re-significación ante el conflicto y las posibilidades que se despliegan desde lo moral y simbólico, para trascender de la intervención denominada "integral". Perpetuar esta mirada, puede extinguir los aportes de procesos estratégicos que involucren la formación y el posicionamiento político, entre otros elementos, los cuales suscitan la reconciliación a partir de iniciativas de reconocimiento y cuidado de sí, para promover el cuidado y bienestar del otro.

- Consolidar una perspectiva de equidad y justicia social como la posibilidad de contar con la garantía de libertades en la esfera de lo íntimo, lo público y lo privado, que permita reconocer a las víctimas del conflicto armado y excombatientes como actores sociales y políticos claves en el proceso de reparación integral, reintegración y consolidación de paz y reconciliación en el posacuerdo, aportando al reconocimiento de sus valores, cultura, capacidades, recursos y potencialidades que deben buscar desplegarse como estrategia de desarrollo humano. Lo anterior se logra a partir de los planes, programas y proyectos que trabajan en esta línea, buscando desde allí trascender esa visión de incapacidad que postulan los procesos asistencialistas y, por el contrario, reivindicar sus voces, sus experiencias, sus historias de vida y su participación social, a partir del reconocimiento político.

- Propiciar intervenciones socio-terapéuticas cimentadas ética y políticamente en la solidaridad, la confianza y la reconciliación que conversen y cuestionen los modelos tradicionales vinculados al estricto seguimiento de lineamientos institucionales, para avanzar en la formación democrática y de participación ciudadana de las víctimas y excombatientes desde lo individual, lo familiar, lo colectivo y lo comunitario, con el fin de incidir en el reconocimiento de sus necesidades sentidas y develar los procesos de organización, construcción de paz, memoria y reconciliación gestados por ellos mismos, a través de la promoción de la co-responsabilidad entre la sociedad civil y el Estado, en perspectiva de participación política en la gestión y evaluación de políticas públicas sociales (Quintero, 2009, p. 8-9). La co-responsabilidad del Estado y sociedad civil puede fortalecer la garantía de los derechos de las víctimas, específicamente el derecho a la reparación en todas sus dimensiones, como reconocimiento del daño que han sufrido individual y colectivamente, si se contextualizan los procesos de intervención desde la atención psico-socio-jurídica integral, de modo que se pueda contemplar la particularidad de la dinámica del conflicto y sus víctimas, y la coresponsabilidad, a partir de la titularidad de derechos, la cual requiere fomentar relaciones interpersonales a partir de acciones comunicativas donde cada sujeto de la familia y de los grupos sociales desarrolle su capacidad de agencia (Galvis, 2011).

- Abordar las intervenciones en lo social desde el paradigma de derechos, desde su garantía en pro de la justicia, la paz y la reparación integral, específicamente en perspectiva del enfoque de derechos, el cual se asume desde la Secretaría Distrital de Planeación (2013) como la garantía que da el Estado de promoción, exigibilidad y ejercicio de los principios y libertades que se otorgan a los sujetos individuales y colectivos, sobre pilares como la igualdad, la diversidad y la equidad. Asumir este enfoque cultiva la garantía de no repetición, con el fin de cooperar en la reivindicación, por ejemplo, del derecho al acceso y tenencia de la tierra, específicamente en poblaciones a las que tradicionalmente les ha sido negada dicha titularidad, haciendo especial énfasis en los grupos de mayor vulnerabilidad y de mayor atención por parte del Estado, aprovechando las orientaciones de las sentencias de la corte suprema de justicia, donde se prioriza la atención a grupos como las mujeres, comunidades afro, niños y niñas con discapacidad, entre otros, sentencias desde donde se generan reivindicaciones de derechos singulares y plurales. 
- Contemplar como un eje de análisis de las intervenciones la perspectiva del enfoque diferencial desde la dimensión socio-cultural, que retoma Simón Gil (2009); dicha perspectiva invita a reconocer y reivindicar la diversidad de nuestro territorio en términos culturales, de género, territoriales, étnicos, de trascurrir vital, entre otros, para lo cual es fundamental generar lecturas micro y macro-sociales del contexto en el cual transita la vida cotidiana de las víctimas del conflicto armado, de los excombatientes y la sociedad civil. La lectura del enfoque diferencial se hace visible en el posicionamiento de la interculturalidad como construcción social, que trasciende la gestación de intercambios desiguales, en los que prima una cultura sobre otra. Dicha perspectiva reconoce el significado político de otras voces que cuestionan el modelo hegemonizado de dominación, y atribuye a la cultura y al lenguaje un papel fundamental como escenario de resistencia a las formas de dominación.

- Consolidar perspectivas diversas en la comprensión de los sujetos y las familias víctimas del conflicto armado, puesto que continuar con la mirada tradicional de familia, centrada en la nuclear como célula básica de la sociedad, deja a un lado la responsabilidad que deben asumir otros actores como el Estado, las instituciones y la misma sociedad civil, que en ocasiones excluyen un vínculo que se puede dar a partir de otras relaciones planteadas por diferentes formas de familiares que existen. La diversidad, entonces, les permite a las familias reconocerse como interlocutoras del Estado en su condición de titular y garante de derechos individuales de sus integrantes, y colectivos como organización social, lo que conlleva a posicionar las familias más allá de la consanguinidad, y a defender su cohesión en relación con la protección como derecho básico de las víctimas del conflicto armado.

- Consolidar desde las expresiones artísticas como la pintura, la música, los relatos orales, las tradiciones folclóricas, entre otras, propuestas metodológicas de intervención socio-terapéutica en escenarios dialógicos y generativos, esos escenarios contemplados por Fried Schnitman (2013) como construcción relacional y polifónica, que se teje dialógicamente a partir de la experiencia conversacional que integra múltiples significados, entre los cuales se ubican los semánticos y los personales. Este proceso de diálogo posibilita reflexionar y movilizar hacia el aprendizaje y la transformación desde posibilidades recursivas de los sujetos. Es así que tal perspectiva socioterapéutica y generativa se integra a los procesos de justicia, reparación y paz, toda vez que involucra la diversidad y riqueza que posee nuestro país, aportando a la consolidación de la resiliencia y la recursividad generadas por las víctimas del conflicto armado (Torres, 2013), quienes han vivido en carne propia los horrores y el dolor ocasionado por un conflicto de tan larga trayectoria. Trabajar desde esta perspectiva aporta a cimentar la memoria histórica y revivir prácticas culturales que se ven afectadas por la re-victimización, la discriminación y la persecución que en algunos entornos de la ciudad padecen las víctimas.

- Configurar desde la socio-terapia con enfoque de acción sin daño una actitud permanente de reflexividad sobre nuestras intervención profesional, proyectando ejercicios de sistematización y de investigación que recuperen los aprendizajes y experiencias como estrategia de producción de conocimiento, en los que según plantea Calvo (2013) se estructuren ejercicios reflexivos en espiral; es decir, procesos de análisis del quehacer, el ser y el saber que se entremezclan y configuran como punto de partida, y a la vez de llegada de la intervención en lo social, desde los cruces entre dimensiones teóricas y metodológicas, entre otras. La reflexividad se enfatiza, entonces, por su capacidad para reconstruir la trayectoria de la intervención, donde emergen componentes que la estructuran, como los mencionados por Mosquera (2013), entre los cuales aparecen la identidad profesional y las propuestas de acción.

Establecer una perspectiva de reflexividad respecto al modo de entender el conflicto armado interno y al modo de intervenir en él, nos mueve a cuestionar cotidianamente los horizontes éticos, políticos y filosóficos de la socio-terapia, a partir de la acción sin daño, enunciados por Bello (2013), puesto que este enfoque se fundamenta en la esencia de la dignidad humana, la libertad y el ejercicio de 
autonomía, dirigidas desde unos mínimos éticos conciliados por la sociedad.

- Finalmente, la mirada de reflexividad de la intervención desde lo socio-terapéutico busca promover hábitos de vida saludables que integren la salud física y mental en los profesionales que trabajan directamente con sujetos afectados por los impactos del conflicto armado, a la luz de los principios filosóficos del autocuidado y la ética del cuidado, para reconocer y minimizar los riesgos psicosociales y emocionales que traen consigo dichos acompañamientos, en los que aparecen altas cargas de tensión, angustia y estrés que pueden generar falta de motivación del profesional, objetivos enfrentados, baja continuidad en los procesos, o impotencia ante la cada vez mayor crisis en la sociedad y menos recursos, como ha enunciado Calvo (2013). De este modo, se contribuye a pensar la salud mental como un derecho subjetivo y colectivo de todos los colombianos, atendiendo a la pregunta por ¿quién cuida al cuidador?

Reconocer que la paz es mucho más que la ausencia de conflictos, y que la reconstrucción del tejido social busca la transformación de la sociedad, es un reto para la intervención en lo social, puesto que la puesta en escena de la socio-terapia requiere ampliar el modo de comprender las emociones en la cotidianidad, como lo sugiere Camps (2011), quien enfatiza la viabilidad de gobernar las emociones como estrategia para la construcción de paz, que supere la hegemónica visión de centrarnos en los hechos negativos que ha dejado el conflicto, para asumir, desde Mosquera (2011), la trasformación de subjetividades sociales desde la creatividad.

Estas perspectivas nos llevan a re-configurar nuestro ser y saber-hacer en la promoción de ejercicios de escucha y participación política de la sociedad civil, principal víctima de este prolongado conflicto. Nos muestran también la oportunidad que tenemos de re-construir nuestro país, potenciando intervenciones en lo social holísticas, históricas, promotoras de diálogos de saberes e intergeneracionales, ecológicas, y éticas. Tales apuestas permitirán que nuestro país configure memoria histórica, y que trabaje hacia la reconciliación, la garantía de los derechos, la no repetición y la dignidad de las víctimas, como camino hacia el perdón y la reconciliación.

Para consolidar el perdón en las esferas públicas y privadas, es relevante aportar desde la intervención en lo social — construida desde la socio-terapiaalternativas innovadoras como el empoderamiento pacifista, planteado por Sandoval (2016), en tanto saber-hacer que incide en la realidad desde propuestas de acción no violentas, fundamento que orienta la reconciliación y configuración de un proyecto de país, que afronta el conflicto y potencia sus capacidades y recursos de diversa índole.

\section{Referencias bibliográficas}

Alayón, N. (2007). Asistencia y asistencialismo¿Pobres controlados o erradicación de la pobreza? Buenos Aires: Lvmen Humanitas.

Bello, M. (2013). Acompañamiento psicosocial a las víctimas en contextos de impunidad. En M. H. Ramírez. (Comp.), La investigación y la práctica en trabajo social (pp. 85-108). Bogotá: Universidad Nacional de Colombia.

Berger P, Luckmann, T. (2003). La construcción social de la realidad. Buenos Aires: Amorrortu Editores.

Calvo, L. (2013). Trabajo social familiar. Transdisciplina y profesión. Buenos Aires: Espacio Editorial.

Camps, V. (2011). El gobierno de las emociones. Madrid: Herder.

Carballeda, A. (2002). La intervención en lo social. Exclusión e integración en los nuevos escenarios sociales. Buenos Aires: Paidos Tramas Sociales.

Carballeda, A. (2010). La intervención en lo social como dispositivo. Una mirada desde los escenarios actuales. Revista de la Escuela Nacional de trabajo social, 6 (1), 46-59. 
Cifuentes, M. (2005). El fortalecimiento de la familia: base para el desarrollo humano y la construcción de relaciones sociales. Bitácora para la interacción psico-social con población afectada por el conflicto armado. Manizales: Universidad de Caldas, Cedat.

Corvalán, R. (1996). Los paradigmas de lo social y las concepciones de la intervención en la sociedad. Montevideo: Universidad de la República de Uruguay.

Fried Schnitman, D. (2013). Prácticas dialógicas generativas en el trabajo con familias. Revista Latinoamericana de Estudios de Familia, 5, 127-159.

Galvis, L. (2011). Pensar la familia hoy. Bogotá: Ediciones Aurora.

Moran Carrillo, J. (2007). Fundamentos del trabajo social: Trabajo social y epistemología. España: Tirant lo Blanch.

Mosquera, C. (2011). Emoción, razón y "proceso civilizatorio": aproximaciones desde los procesos de atención psicosocial de personas desplazadas por el conflicto armado colombiano. En L. Arango \& P. Molinier. (Ed.), El trabajo y la ética del cuidado (pp. 275-294). Medellín: La Carreta editores.

Mosquera, C. (2013). Pluralismos epistemológicos: hacia la valoración teórica de los saberes de acción. Una reflexión desde la intervención social a la población afrocolombiana desplazada. En M. Ramírez. (Ed.), La investigación y la práctica en trabajo social (pp. 53-84). Bogotá: Universidad Nacional de Colombia.

Muñoz, F. (2011). La paz imperfecta ante un universo en conflicto. Granada: Universidad de Granada.

Pineda, E. De Alvarado, E. (2008). Metodología de la investigación. Washington: Organización Panamericana de la Salud.
Quintero, A. (octubre, 2009). Trabajo social en los nuevos escenarios de infancia, adolescencia y familia. En Memorias 1 XIX. Seminario Latinoamericano de Escuelas de Trabajo Social. El trabajo social en la coyuntura latinoamericana: desafios para su formación, articulación y acción profesional. Ponencia llevada a cabo en la Universidad Católica Santiago de Guayaquil. Guayaquil, Ecuador.

Rozas, M. (2001). La Intervención profesional en relación a la cuestión social: el caso del trabajo social. Buenos Aires: Editorial Espacio.

Sampieri, R. (2010). Metodología de la investigación. Lima: Mc Graw Hill.

Sandoval, E. (2016). Educación para la paz integral. Memoria, interculturalidad y decolonialidad. Bogotá: Arfo Editores e impresores LTDA.

Secretaría Distrital de Planeación. (2013). Documento de trabajo: Lineamientos para el diseño e implementación. Observatorio social para las familias del Distrito. Bogotá: Alcaldía Mayor de Bogotá.

Torres, F. (2013). Intervención profesional desde la consultoría con enfoque resiliente en familias víctimas de conflicto armado. Revista Tendencias y Retos, 18 (1), 33-48.

Vasco, E. (1990). Tres estilos de trabajo en las ciencias sociales: Comentarios a propósito del artículo "Conocimiento e Interés" de Jürgen Habermas. Bogotá: CINEP Centro de Investigación y educación popular.

Vives, M. (2015). Confianza: propuesta de un modelo teórico sobre su génesis y consolidación. Bogotá: Ediciones Unisalle. 